Instituto Internacional de Investigación y Desarrollo Tecnológico Educativo INDTEC, C.A. DOI: https://doi.org/10.29394/Scientific.issn.2542-2987.2020.5.E.4.90-109

OAI-PMH: http://www.indteca.com/ojs/index.php/Revista Scientific/oai

Artículo Original / Original Article

\title{
Mujeres víctimas de violencia de género en Ecuador: redes de apoyo y estrategias de afrontamiento
}

Autores: Luís Gabriel Benalcázar Mancero Universidad Internacional SEK, UISEK lgbenalcazar.mpp@uisek.edu.ec

Quito, Ecuador https://orcid.org/0000-0003-0136-2209

Paulo César Damián Carrión Universidad Internacional SEK, UISEK pcdamian.mpp@uisek.edu.ec

Quito, Ecuador https://orcid.org/0000-0002-1756-5476

Pamela Valeria Yarad Jeada Universidad Internacional SEK, UISEK vale.yarad@gmail.com

Quito, Ecuador

https://orcid.org/0000-0002-5040-4324

\section{Resumen}

La Encuesta Nacional sobre Relaciones Familiares y Violencia de Género contra las Mujeres realizada por el Instituto Nacional de Estadística y Censos (INEC, 2019): evidencia que un $64,9 \%$ de las mujeres han sufrido algún episodio de violencia a lo largo de su vida. El presente trabajo tiene como objetivo analizar las redes de apoyo y las estrategias de afrontamiento de mujeres que han sufrido violencia de género y que han acudido a la Defensoría Pública, para ello se realizaron encuestas a 75 mujeres de distintas edades. La investigación se llevó a cabo en el marco del proyecto de investigación que lleva adelante la Universidad Internacional SEK (UISEK), en la cual se planteó a las participantes una matriz de 12 problemas y 12 personas a las que podrían recurrir en caso de necesitar ayuda. Como resultado se observó que la mayor red de apoyo se centra en el ámbito cercano (madre, hermana, mejor amiga, psicóloga, padre), estas redes pueden constituirse en recursos para el afrontamiento a la violencia experimentada.

Palabras clave: violencia doméstica; género humano; encuesta.

Cómo citar este artículo:

Benalcázar, L., Damián, P., \& Yarad, P. (2020). Mujeres víctimas de violencia de género en Ecuador: redes de apoyo y estrategias de afrontamiento. Revista Scientific, 5(Ed. Esp.), 90-109, e-ISSN: 2542-2987. Recuperado de: https://doi.org/10.29394/Scientific.issn.2542$\underline{\text { 2987.2020.5.E.4.90-109 }}$

Fecha de Recepción: 04-04-2020
Fecha de Aceptación: 26-06-2020
Fecha de Publicación: 05-08-2020 
OAl-PMH: http://www.indteca.com/ojs/index.php/Revista_Scientific/oai

Artículo Original / Original Article

\section{Women victims of gender violence in Ecuador: support networks and coping strategies}

\begin{abstract}
In Ecuador, in the last survey from National Institute of Statistics and Census, INEC (2019) say that $64.5 \%$ of women has been some violence episode in them lives, as physical, sexual, physiologic, patrimonial and gynecologic. This work has a quantitative approach, though survey and analyzed support networks and handle strategies from 75 women of different ages who have been victims of violence and who have filed the complaint with the Public Defender, whose autonomous body of the Judicial Function guarantees access to justice for people in vulnerable situations. This survey was carried out in the research project carried out by SEK International University, in which participants were presented with a matrix of 12 problems and 12 people to whom they could turn if they needed help. As a result, it was observed that the largest support network is focused on the immediate environment (mother, sister, best friend, psychologist, father), these networks can become resources for coping with the violence experienced.
\end{abstract}

Keywords: domestic violence; human beings; survey.
How to cite this article:

Benalcázar, L., Damián, P., \& Yarad, P. (2020). Women victims of gender violence in Ecuador: support networks and coping strategies. Revista Scientific, 5(Ed. Esp.), 90-109, e-ISSN: 25422987. Recovered from: https://doi.org/10.29394/Scientific.issn.2542-2987.2020.5.E.4.90-109
Date Acceptance:

26-06-2020
Date Publication:

05-08-2020 
OAI-PMH: http://www.indteca.com/ojs/index.php/Revista_Scientific/oai

Artículo Original / Original Article

\section{Introducción}

La violencia en contra de la mujer de acuerdo a las conceptualizaciones expuestas por las Naciones Unidas (1995): en la IV Conferencia Mundial sobre la Mujer en Beijing, se define como: cualquier acto perpetrado con violencia en contra de una mujer por motivos de sexo, que deje como resultado secuelas de cualquier tipo, incluyendo lesiones físicas, daño psicológico y/o sexual, o a que a su vez este tipo de agresiones generen sufrimiento en la victima; dichos actos violentos suelen incluir amenazas, coacción y privación de la libertad.

La violencia no se limita a un contexto social, cultural o familiar; tampoco a una etnia o un subgrupo especifico de mujeres en una sociedad, se ha convertido en un problema endémico que afecta a todas las mujeres en el mundo. Según los datos estadísticos emitidos por la Organización Mundial de la Salud (OMS, 2005): recopilados de estudios realizados a nivel multipaís estima que los índices de violencia registrados por parte de la pareja oscilaban entre el $24 \%$ y el $53 \%$, que acarreaban golpes y que podían llevar a la muerte de la mujer.

El Observatorio de Igualdad de Género de América Latina y el Caribe de la Comisión Económica para América Latina y el Caribe (CEPAL, 2018): señala que en los 15 países analizados al menos 3.287 mujeres han sido víctimas de feminicidio o femicidio, en lo que respecta a Ecuador, hasta el año 2017 se registraron 104 femicidios/feminicidios con una tasa de 1.3 asesinatos por cada 100.000 mujeres.

En el Ecuador, la última Encuesta Nacional sobre Relaciones Familiares y Violencia de Género contra las Mujeres del Instituto Nacional de Estadística y Censos (INEC, 2019a): corrobora los datos a nivel regional, tomando en cuenta que 65 de cada 100 mujeres en el Ecuador, han experimentado por lo menos un hecho de algún tipo de violencia en alguno de los distintos ámbitos a lo largo de su vida y 32 de cada 100 mujeres en los últimos 12 meses. En los datos de la encuesta, cabe destacar que no se presentan diferencias 
significativas en lo que respecta al nivel educativo o económico. Sin embargo, el factor etnicidad si indica ciertas diferencias, por ejemplo, las mujeres afroecuatorianas han experimentado mayor violencia a lo largo de su vida $71,8 \%$. En lo referente al ámbito donde se genera la violencia, cabe destacar que existe una prevalencia de violencia en el ámbito doméstico o de pareja, a lo largo de su vida un $42,8 \%$ de las mujeres han experimentado violencia por parte de sus parejas, y en los últimos 12 meses un $18 \%$.

De este porcentaje, el Instituto Nacional de Estadística y Censos (INEC, 2019b): estima que un 40,8 ha sido del tipo psicológica, un $53,8 \%$, un $25 \%$ física, un 14,5\% patrimonia, un 19,5\% sexual. Estos episodios son justificados en la mayoría de los casos por los roles de género que normalizan la violencia contra la mujer. A nivel nacional, expone que el $27 \%$ de las mujeres creen que una buena esposa debe obedecer a su esposo en todo lo que él ordene, un $45 \%$ afirman que las mujeres deben ser las responsables de las tareas de la casa, cuidado de los hijos/as, de las personas enfermas y ancianas, un $57 \%$ señalan que las mujeres deben actuar y vestirse recatadamente para no provocar.

En el Boletín Estadístico de la Defensoría Pública del Ecuador (2018): entidad autónoma del Poder Judicial, que brinda asesoría jurídica a personas vulnerables señala que entre los principales tipos de delito se encuentran: las contravenciones de violencia contra la mujer o miembros del grupo familiar 2.883 casos que representan el $27,2 \%$; Delitos contra la integridad sexual y reproductiva, 2.003 denuncias 18,9\%; Delitos de violencia contra la mujer o miembros del núcleo familiar, 1.682 demandas $15,8 \%$.

Ante la gravedad de esta problemática y lo impactante de los datos recopilados por los organismos competentes como la Oficina de Drogas y Crimen de las Naciones Unidas, United Nations Office on Drugs and Crime (UNODC, 2010): la mayoría de naciones a nivel mundial han tomado medidas, en América Latina, 18 países incluyendo Ecuador, han modificado sus leyes 
OAI-PMH: http://www.indteca.com/ojs/index.php/Revista_Scientific/oai

Artículo Original / Original Article

para prevenir y sancionar estos actos. En función a estos antecedentes, esta investigación tiene como base la aproximación ecológica, en la que destaca que toda acción está influida por el entorno en el que se desarrollan los sujetos.

Desde la perspectiva de Torrico, Santín, Andrés, Menéndez y López (2002): el modelo ecológico, se basa en una primera instancia en el microsistema que es el nivel de interacción más cercano al individuo, constituido por la familia, amigos y demás persona con vínculos cercanos; en éste es donde la persona va construyendo su percepción acerca de la realidad, además es donde recibe mayor apoyo emocional. En el segundo nivel se encuentran los mesosistemas que representan la vinculación e interacción de dos microsistemas en los que se relaciona el individuo, estos pueden ser: familia-escuela, familia-trabajo y demás interrelaciones posibles.

La tercera instancia se refiere a los exosistemas, en cuyo entorno el individuo no participa como un sujeto activo, sin embargo, los hechos que suceden en estos sistemas logran afectar en menor o mayor grado a los entornos en los que sí está vinculado. En última instancia está el macrosistema que se trata de un entorno más distante, pero que engloba a los demás sistemas existentes, en éste se sitúan la cultura, la religión, la historia y las instituciones.

La Organización Panamericana de la Salud (OPS), realizó en conjunto con la Organización Mundial de la Salud (OMS, 2002): un estudio mundial sobre la violencia y la salud a pedido desde la perspectiva ecológica, para investigar sobre los factores sociales, familiares y personales como un recurso de protección o a su vez riesgo en la problemática de violencia de género, se lograron identificar diversos factores implicados en los contextos sociales, destaca los siguientes:

- Factores biológicos: caracteres demográficos, trastornos psicológicos; adicciones y dependencias, antecedentes de agresividad. 
- Factores familiares: familia, amigos, la pareja y los compañeros.

- Factores comunitarios: escuela, el trabajo, el vecindario.

- Factores de carácter general: relacionados a la estructura social. En este nivel, se consideran las políticas públicas en salud y educación.

Para Buesa y Calvete (2013): la violencia de género en contra de la mujer y la función del apoyo social es una temática cuyo análisis se debe enfocar desde los cuatro sistemas propuestos por Bronfenbrenner (1987): los cuales son: microsistema, mesosistema, macrosistema y exosistema, destacándose entre los demás el microsistema, pues dentro de este se van dando un sinnúmero de dinámicas interpersonales que la mujer va experimentado en un contexto especifico, con particularidades ambientales en las que se puede situar al ambiente familiar y vínculos de amistad.

De acuerdo con Guerra y Dierkhising (2011): la comunidad es un sistema macrosocial de interacción interpersonal donde también se evidencia y normaliza la violencia, por ejemplo, el acoso en las calles, la cosificación, el irrespeto del espacio personal de la mujer en los medios de transporte, etc. Como dice Acevedo (2012): otro de los sistemas en donde se evidencia claramente la violencia en contra de la mujer es el área laboral-académica, correspondientes a un mesosistema, donde estadísticamente se demuestra que las agresiones suelen darse en mayor porcentaje en contra de las mujeres, siendo estas propiciadas generalmente por una persona de jerarquía superior inmediata.

La violencia en los exosistemas asociados a los medios de comunicación es otro de los aspectos influyentes en la normalización de esta. Mencionando a Galdames y Arón (2007): en la actualidad existe una excesiva exposición de la sociedad a medios de información y comunicación masiva con contenidos violentos en noticiarios, periódicos, redes sociales e incluso 
OAI-PMH: http://www.indteca.com/ojs/index.php/Revista_Scientific/oai

\section{Artículo Original / Original Article}

discursos políticos que se suma a la legitimación de la violencia.

Cabe destacar según Lin, Dean y Ensel (1986): por tal razón, la importancia de las redes de apoyo, cuando una mujer se encuentra inmersa dentro de un círculo recurrente de violencia, tomando a estas como aquellos vínculos emocionales cercanos que percibe un individuo y que obtiene de la comunidad, familia y amigos, que generan en la persona una sensación de confianza y seguridad. De acuerdo con Hadeed y El-Bassel (2006): las mujeres al tomar conciencia de las posibles repercusiones del abuso sobre su integridad, adoptan estrategias de afrontamiento ante la violencia, las mismas que van desde el hecho de separase de la relación, hasta pedir ayuda acudiendo a su red de apoyo más cercana o denunciar los acontecimientos ante las instituciones pertinentes.

Interpretando a Belknap, Melton, Denney, Fleury-Steiner y Sullivan (2009): En algunos casos las mujeres se perpetúan dentro del círculo de violencia sin distanciarse de su agresor siendo las más vulnerables aquellas que percibían tener menor apoyo social por parte de sus vínculos familiares y de amistad y menor respaldo aún a nivel institucional.

En este contexto, Moral de la Rubia, López, Díaz y Cienfuegos (2011): destacan la importancia del afrontamiento en lo referente a los conflictos de pareja, alegando que un adecuado afrontamiento ante esta problemática evita que esta situación se convierta en crónica; de igual manera, Díaz y Sánchez (2002): recalcan la relevancia de un afrontamiento eficaz, como un factor que podría prevenir circunstancias de violencia recurrentes.

Señalando a Márquez (2006): el afrontamiento no es más que la gestión de dichas situaciones de estrés y tensión que suceden cuando una persona ha sido víctima de una circunstancia traumática en su vida. En este mismo orden de ideas, Frydenberg y Lewis (2000): argumentan que existen dos tipos de afrontamiento, el general y el específico, el primero son aquellas predisposiciones habituales con las que debe lidiar el ser humano en cualquier 
situación cotidiana, mientras que la segunda hace referencia a todas aquellas estrategias que se emplean para hacerle frente a una demanda particular generadora de estrés.

Conforme a Castaño y León del Barco (2010); y Folkman (2011): el afrontamiento son aquellas gestiones cognitivas y conductuales que va a implementar el ser humano con la finalidad de enfrentarse a eventos de estrés interno o externo, éstas pueden ser cambiantes y adaptables a cada situación. Interpretando a Rodríguez-Marín, Pastor y López-Roig (1993): para que las estrategias de afrontamiento sean exitosas, éstas dependerán de los recursos de afrontamiento con los que cuenten los sujetos y que son los medios que un individuo tiene a su alcance para enfrentar una situación conflictiva.

Estos recursos antes mencionados se clasifican de la siguiente manera: culturales, se obtienen del entorno cultural de la persona; físicos, relacionado a su estado de salud; psicológicos, asociados a la configuración cognitiva, el estado afectivo y a los esquemas de comportamiento; estructurales, son los roles y las redes socialmente estipulados; y sociales en base a las relaciones interpersonales. En conformidad con Huijts, Kleijn, van Emmerik, Noordhof y Smith (2012); y Luthar y Cicchetti (2000): cuando la mujer víctima de violencia afronta su situación implementa estas y otras estrategias que le permitirá adaptarse a circunstancias adversas que forman parte del proceso de resiliencia.

Por consiguiente, Carver, Scheier y Weintraub (1989): plantean un afrontamiento de tres tipos basados en la emoción, centrado en el problema, centrado en la evitación, estas ideas fueron tomadas por Londoño, Henao, Puerta, Posada, Arango y Aguirre (2006): quienes proponen 12 estrategias de afrontamiento basadas en resolución de conflictos, apoyo social, resiliencia, doctrina religiosa, contención emocional, intervención profesional, respuesta agresiva, contención cognitiva, reevaluación positiva, expresión de dificultades, negación, y finalmente autonomía. 
OAI-PMH: http://www.indteca.com/ojs/index.php/Revista_Scientific/oai

\section{Artículo Original / Original Article}

En base a lo expuesto, este artículo pretende mostrar las redes de apoyo señaladas por las mujeres víctimas de violencia y posibles escenarios de afrontamiento en miras de que tengan las herramientas necesarias para romper el círculo de la violencia.

\section{Materiales y métodos}

El presente trabajo se desarrolló desde una perspectiva cuantitativa de tipo descriptivo, mediante la realización de una encuesta a 75 mujeres que interpusieron una demanda por violencia de género en la Defensoría Pública y que han salido del círculo de la violencia, el instrumento fue desarrollado por investigadores de la Universidad de Guadalajara (México) y aplicado en el proyecto de investigación de la Universidad Internacional SEK (UISEK) en Quito, el mismo que está diseñado para identificar la red de apoyo de mujeres que han sufrido violencia de género por parte de su pareja y la confianza que tienen en los sistemas micro-meso-macro. La mayor parte de la muestra está conformada en su mayoría por mujeres de entre 26 a 45 años, el perfil socioeconómico es diverso, con mayor representación de amas de casa y estudiantes, que son quienes en su mayoría denuncian. Un aspecto por destacar es el nivel de estudios, un $25 \%$ de las encuestadas tienen título de licenciatura o maestría.

Consecuentemente, Herrera (2018): expone que el desarrollo de nuevas perspectivas de investigación demanda que los abordajes metodológicos se adapten a las distintas problemáticas a ser indagadas desde la sociedad.

\section{Resultados}

En la tabla 1, se observan los resultados que apuntan a las redes de apoyo que se conforman en torno al microsistema de las denunciantes, la madre es el principal punto de referencia para las mujeres en todos los 
aspectos, seguido de los hermanos, indistintamente del sexo de los mismos, en las preguntas más relacionadas a búsqueda de ayuda en el ámbito económico, la madre, el padre y la hermana son la red a la que más se acudiría. La psicóloga, cabe destacar que en este caso se habla en femenino, se observa que es la persona en la cual las víctimas dicen que acudirían en caso de necesitar apoyo, en caso de presentar problemas de salud física o daños emocionales, cuando tienen problemas de ánimo, para contar sus problemas de pareja y para pedir consejo, en este caso se observa que las denunciantes prefieren esa ayuda externa a su círculo más cercano cuando tienen problemas del tipo emocional, los cuales necesitan ser gestionados, siendo una forma de afrontamiento a los problemas del tipo psicológico.

Tabla 1. Matriz de problemas y de personas pertenecientes a las redes de apoyo.

\begin{tabular}{|c|c|c|c|c|c|c|c|c|c|c|c|c|c|}
\hline & $\begin{array}{l}\text { Acudir en caso } \\
\text { de necesitar } \\
\text { apoyo }\end{array}$ & $\begin{array}{l}\text { Problemas } \\
\text { de salud } \\
\text { fisica, } \\
\text { daños } \\
\text { lesiones }\end{array}$ & $\begin{array}{c}\text { Problemas, } \\
\text { estado de } \\
\text { ánimo }\end{array}$ & $\begin{array}{c}\text { Sentido } \\
\text { que no } \\
\text { vales lo } \\
\text { suficiente }\end{array}$ & $\begin{array}{l}\text { Falta de } \\
\text { dinero } \\
\text { con } \\
\text { urgencia }\end{array}$ & $\begin{array}{c}\text { Has } \\
\text { contado a } \\
\text { alguien lo } \\
\text { que te } \\
\text { pasa con } \\
\text { tu pareja }\end{array}$ & $\begin{array}{c}\text { Ha estado } \\
\text { en peligro } \\
\text { tu vida }\end{array}$ & $\begin{array}{l}\text { Has } \\
\text { necesitado } \\
\text { consejos }\end{array}$ & $\begin{array}{c}\text { Apoyo } \\
\text { para } \\
\text { conseguir } \\
\text { trabajo }\end{array}$ & $\begin{array}{l}\text { Apoyo para } \\
\text { cuidar a tus } \\
\text { hijos }\end{array}$ & $\begin{array}{l}\text { Acompañe } \\
\text { a poner la } \\
\text { denuncia }\end{array}$ & $\begin{array}{l}\text { Necesidad } \\
\text { de refugio }\end{array}$ & $\begin{array}{c}\text { Acompañar a } \\
\text { recibir } \\
\text { atención } \\
\text { médica o } \\
\text { psicológica }\end{array}$ \\
\hline Padre & $36,0 \%$ & $14,7 \%$ & $9,3 \%$ & $5,3 \%$ & $20,0 \%$ & $8,0 \%$ & $10,7 \%$ & $6,7 \%$ & $16,0 \%$ & $9,3 \%$ & $10,7 \%$ & $17,3 \%$ & $10,7 \%$ \\
\hline Madre & $62,7 \%$ & $41,3 \%$ & $33,3 \%$ & $28,0 \%$ & $42,7 \%$ & $41,3 \%$ & $40,0 \%$ & $36,0 \%$ & $33,3 \%$ & $40,0 \%$ & $38,7 \%$ & $45,3 \%$ & $41,3 \%$ \\
\hline Abuelo & $8,0 \%$ & $1,3 \%$ & - & - & - & - & - & $1,3 \%$ & - & - & $1,3 \%$ & - & - \\
\hline Abuela & $8,0 \%$ & $1,3 \%$ & - & $1,3 \%$ & - & $1,3 \%$ & - & $1,3 \%$ & $1,3 \%$ & - & $0,0 \%$ & $1,3 \%$ & - \\
\hline Hermano & $26,7 \%$ & $8,0 \%$ & $8,0 \%$ & $4,0 \%$ & $12,0 \%$ & $13,3 \%$ & $13,3 \%$ & $9,3 \%$ & $10,7 \%$ & $16,0 \%$ & $13,3 \%$ & $8,0 \%$ & $6,7 \%$ \\
\hline Hermana & $42,7 \%$ & $22,7 \%$ & $20,0 \%$ & $14,7 \%$ & $20,0 \%$ & $26,7 \%$ & $17,3 \%$ & $16,0 \%$ & $17,3 \%$ & $22,7 \%$ & $18,7 \%$ & $21,3 \%$ & $18,7 \%$ \\
\hline Hijo & $25,3 \%$ & $13,3 \%$ & $6,7 \%$ & $5,3 \%$ & $5,3 \%$ & $9,3 \%$ & $8,0 \%$ & $5,3 \%$ & $6,7 \%$ & $1,3 \%$ & $10,7 \%$ & $5,3 \%$ & $10,7 \%$ \\
\hline Hija & $21,3 \%$ & $10,7 \%$ & $6,7 \%$ & $10,7 \%$ & $5,3 \%$ & $9,3 \%$ & $8,0 \%$ & $4,0 \%$ & $5,3 \%$ & $4,0 \%$ & $6,7 \%$ & $5,3 \%$ & $10,7 \%$ \\
\hline Nieto & $1,3 \%$ & - & - & - & - & - & - & - & - & - & - & - & - \\
\hline Nieta & $2,7 \%$ & - & - & - & - & - & - & - & - & - & - & - & - \\
\hline Vecina & $16,0 \%$ & $16,0 \%$ & $4,0 \%$ & $1,3 \%$ & $4,0 \%$ & $5,3 \%$ & $5,3 \%$ & $2,7 \%$ & $5,3 \%$ & $4,0 \%$ & $4,0 \%$ & $4,0 \%$ & $4,0 \%$ \\
\hline Vecino & $4,0 \%$ & $4,0 \%$ & $1,3 \%$ & - & $1,3 \%$ & $0,0 \%$ & $1,3 \%$ & - & - & $1,3 \%$ & - & $1,3 \%$ & - \\
\hline Psicológo & $13,3 \%$ & $13,3 \%$ & $8,0 \%$ & $8,0 \%$ & - & $6,7 \%$ & $4,0 \%$ & $8,0 \%$ & - & & $1,3 \%$ & - & - \\
\hline Psicológa & $61,3 \%$ & $61,3 \%$ & $46,7 \%$ & $37,3 \%$ & $2,7 \%$ & $48,0 \%$ & $21,3 \%$ & $48,0 \%$ & $5,3 \%$ & $1,3 \%$ & $2,7 \%$ & $4,0 \%$ & $2,7 \%$ \\
\hline $\begin{array}{l}\text { Homeópata/ } \\
\text { naturista }\end{array}$ & $1,3 \%$ & $1,3 \%$ & - & - & - & - & - & - & - & - & - & - & - \\
\hline Pareja & $13,3 \%$ & $13,3 \%$ & $2,7 \%$ & $1,3 \%$ & $8,0 \%$ & $4,0 \%$ & $2,7 \%$ & $4,0 \%$ & $2,7 \%$ & $4,0 \%$ & $4,0 \%$ & $2,7 \%$ & $5,3 \%$ \\
\hline $\begin{array}{l}\text { Sacerdote/ } \\
\text { consejero }\end{array}$ & $21,3 \%$ & $21,3 \%$ & $10,7 \%$ & $2,7 \%$ & $4,0 \%$ & $10,7 \%$ & $6,7 \%$ & $13,3 \%$ & $2,7 \%$ & $2,7 \%$ & $1,3 \%$ & $1,3 \%$ & $2,7 \%$ \\
\hline Policía & $21,3 \%$ & $21,3 \%$ & - & - & - & $5,3 \%$ & $13,3 \%$ & $4,0 \%$ & - & - & $5,3 \%$ & - & $2,7 \%$ \\
\hline Maestro & $8,0 \%$ & $8,0 \%$ & $1,3 \%$ & - & - & $1,3 \%$ & $1,3 \%$ & $1,3 \%$ & $1,3 \%$ & - & $1,3 \%$ & - & - \\
\hline Maestra & $9,3 \%$ & $9,3 \%$ & - & $1,3 \%$ & $1,3 \%$ & $1,3 \%$ & - & $1,3 \%$ & - & $1,3 \%$ & - & - & - \\
\hline
\end{tabular}

Fuente: Las Autoras (2020). 
OAI-PMH: http://www.indteca.com/ojs/index.php/Revista_Scientific/oai

\section{Artículo Original / Original Article}

En la matriz de 12 problemas que se plantea a las encuestadas, ellas señalan que pueden recurrir en su mayoría a la madre $(62,7 \%)$, la hermana $(42,7 \%)$, la mejor amiga $(42,7 \%)$, la psicóloga $(61,3 \%)$ y el padre $(36 \%)$. Se partió de la pregunta ¿A quién acudirías en caso de? y se les presentó una serie de personas relacionadas a su entorno que podrían ser parte de la red de apoyo. En la Tabla 1 se presentan estos resultados.

Otro aspecto importante a recalcar, es la falta de confianza que tienen las mujeres víctimas en otras personas de su entorno, por ejemplo, dentro del sistema institucional, pocos acudirían a su maestro/a o al policía. Algunas de ellas acudirían a la vecina, y sucede lo mismo que con la psicóloga, en estos casos procuran acudir a un soporte femenino, ya que es probable que sientan mayor empatía por parte de otras mujeres.

Las mujeres que sufren episodios de violencia tienen cada vez menos confianza y sienten menos cercanía de quien les rodea, es así como la mayoría de las veces acuden a su círculo más cercano. De modo que, los otros miembros de su entorno como vecinos, sacerdote/consejero, médico, etc., no son parte activa de esa red de apoyo.

Un $76 \%$ de las participantes en el estudio tienen hijos, con una media de 2,16 hijos. Las edades de los hijos fluctúan entre los 10 meses hasta los 43 años. Asimismo, la media del tiempo de relación con el demandado (desde el noviazgo) es de 13 años, de modo que se puede presumir de episodios de violencia "normalizados".

Al momento de enseñarles a las mujeres cuáles son los comportamientos violentos más habituales de sus parejas en contra de ellas se evidencia en su mayoría episodios relacionados a violencia psicológica y patrimonial, las víctimas aseguran que sus parejas les hacen sentirse inferiores, no las valoran intelectualmente o las hacen sentirse inútiles o poco atractivas. Además, se molestan al proveer recursos para el hogar y tienden a ser celosos, esto último asociado al ideal del "amor romántico" 
El estudio apunta a que un $74 \%$ de mujeres confían mucho en la madre, un $70 \%$ en la psicóloga (mujer) y un $60 \%$ en el psicólogo (hombre), un $56 \%$ en la hermana frente a un $50 \%$ en el hermano. La confianza en el padre es de un $37 \%$, mucho menor a todo el grupo familiar. Esto puede deberse a la imagen masculina reflejada en el maltratador y que genera esa desconfianza en la mujer. De modo que, se observa las redes de apoyo se centran en el entorno más familiar y cotidiano de la víctima.

Finalmente, cuando se les preguntó a las mujeres: si estuvieras en una seria crisis personal, ¿Cuántas personas crees que tratarían de ayudarte?, un $69,3 \%$ afirma que podría contar con $2-5$ personas y un $18,7 \%$ en sólo 1 persona, esto hace notorio que las redes de confianza son cada vez menores, por lo cual las mujeres empiezan a desarrollar estrategias de afrontamiento basadas en sus necesidades para salir de los problemas relacionados con la violencia, tomando como referencia, que muchas de esas mujeres son madres y tienen a cargo la crianza y cuidado de sus hijos.

\section{Conclusiones}

Las estrategias de afrontamiento deben centrarse en dotar a la víctima de recursos que en una primera fase se concentren en fortalecer las redes de apoyo, brindando a la víctima un soporte familiar, colectivo y estatal para romper el círculo de la violencia.

Además, una vez la mujer se encuentra consciente de que está experimentando esta situación, desde la psicoterapia, y otras disciplinas como el trabajo social, las leyes, la medicina, etc., generar una estrategia integral que dote de recursos a estas mujeres, de modo de que no tengan la obligación de volver con su maltratador. En el artículo se observó que las redes de apoyo conformadas por la madre, hermana, psicóloga, el padre y mejor amiga han sido las de mayor prevalencia, constituyéndose en potenciales estrategias de afrontamiento dentro del grupo de mujeres participantes en el estudio, ya que 
perciben que en el apoyo social y profesional encontrarían recursos para hacerle frente a la problemática de violencia experimentada, pues en estos vínculos encuentran soporte emocional, económico, patrimonial y físico; estos factores antes mencionados han sido fundamentales para que las mujeres encuestadas logren salir del círculo de violencia.

Existen otras posibilidades para establecer redes de apoyo a nivel macro, meso y microsocial, las mismas que están situadas dentro del ambiente familiar, social, comunitario y gubernamental, sin embargo, el grupo en estudio no ha logrado establecer vínculos significativos de confianza que les ayuden a afrontar la problemática de violencia de género.

Dentro del grupo de mujeres que formaron parte del estudio las redes de apoyo han sido un factor fundamental para el afrontamiento de la problemática de violencia vivenciada, sin embargo, estas redes de confianza son muy reducidas, pues el mayor porcentaje de las mujeres encuestadas sitúa su red de apoyo en un máximo de 5 personas en el mejor de los casos y otro porcentaje elevado considera que ante una crisis situacional solamente 1 persona acudiría para ayudarlas, datos que reflejan la necesidad de crear conciencia colectiva acerca de la importancia de brindar apoyo social a la mujer víctima de violencia de género para un mejor afrontamiento de esta problemática.

\section{Referencias}

Acevedo, D. (2012). Violencia laboral, género y salud. Trabajadoras y trabajadores de la manufactura. Salud de los Trabajadores, 20(2), 167-177, ISSN: 1315-0138, e-ISSN: 2665-0215. Recuperado de: http://servicio.bc.uc.edu.ve/multidisciplinarias/saldetrab/index.htm Belknap, J., Melton, H., Denney, J., Fleury-Steiner, R., \& Sullivan, C. (2009). The Levels and Roles of Social and Institutional Support Reported by Survivors of Intimate Partner Abuse. Feminist Criminology, 4(4), 
OAI-PMH: http://www.indteca.com/ojs/index.php/Revista_Scientific/oai

Artículo Original / Original Article

377-402, e-ISSN: 1557-086X. Recovered from:

https://doi.org/10.1177/1557085109344942

Buesa, S., \& Calvete, E. (2013). Violencia contra la mujer y síntomas de depresión y estrés postraumático: el papel del apoyo social. International Journal of Psychology and Psychological Therapy, 13(1), 31-45, e-ISSN: 1577-7057. Recuperado de:

https://www.ijpsy.com/volumen13/num1/345.html

Bronfenbrenner, U. (1987). La ecología del desarrollo humano. Madrid, España: Ediciones Paidós.

Carver, C., Scheier, M., \& Weintraub, J. (1989). Assessing coping strategies: A theoretically based approach. Journal of Personality and Social Psychology, 56(2), 267-283, e-ISSN: 1939-1315. Recovered from: https://doi.org/10.1037/0022-3514.56.2.267

Castaño, E., \& León del Barco, B. (2010). Estrategias de afrontamiento del estrés y estilos de conducta interpersonal. International Journal of Psychology and Psychological Therapy, 10(2), 245-257, e-ISSN: 15777057. Recuperado de:

https://www.ijpsy.com/volumen10/num2/260.html

CEPAL (2018). Feminicidio. New York, Estados Unidos: Observatorio de Igualdad de Género de América Latina y el Caribe del CEPAL Naciones Unidas. Recuperado de:

https://oig.cepal.org/es/indicadores/feminicidio

Defensoría Pública del Ecuador (2018). Boletín Estadístico. Quito, Ecuador: Dirección Nacional de Investigaciones Aplicadas de la Defensoría Pública del Ecuador. Recuperado de:

https://www.defensoria.gob.ec/wp-

content/uploads/2019/06/boletinestadistico2018.pdf

Díaz, R., \& Sánchez, R. (2002). Psicología del amor: Una visión integral de la relación de pareja. México: Editorial. Miguel Ángel Porrúa. 
Folkman, S. (2011). The Oxford Handbook of Stress, Health, and Coping. ISBN: 0195375343, ISBN: 9780195375343. New York, United States: Oxford University Press.

Frydenberg, E., \& Lewis, R. (2000). ACS: escalas de afrontamiento para adolescentes. 3ra. edición, ISBN: 84-7174-636-0. Madrid, España: Tea ediciones.

Galdames, S., \& Arón, A. (2007). Construcción de una Escala Para Medir Creencias Legitimadoras de Violencia en la Población Infantil. Psykhe, 16(1), 15-25, e-ISSN: 0717-0297. Recuperado de: http://dx.doi.org/10.4067/S0718-22282007000100002

Guerra, N., \& Dierkhising, C. (2011). Los efectos de la violencia comunitaria sobre el desarrollo del niño. En: Tremblay RE, Boivin M, Peters $\mathrm{RDeV}$, eds. Tremblay RE, ed. tema. Canadá, Estados Unidos: Enciclopedia sobre el Desarrollo de la Primera Infancia.

Hadeed, L., \& El-Bassel, N. (2006). Social Support Among Afro-Trinidadian Women Experiencing Intimate Partner Violence. Violence Against Women, 12(8), 740-760, e-ISSN: 1552-8448. Recovered from: https://doi.org/10.1177/1077801206291562

Herrera, J. (2018). Las prácticas investigativas contemporáneas. Los retos de sus nuevos planteamientos epistemológicos. Revista Scientific, 3(7), 6-15, e-ISSN: 2542-2987. Recuperado de: https://doi.org/10.29394/Scientific.issn.2542-2987.2018.3.7.0.6-15

Huijts, I., Kleijn, W., van Emmerik, A., Noordhof, A., \& Smith, A. (2012). Dealing with man-made trauma: The relationship between coping style, posttraumatic stress, and quality of life in resettled, traumatized refugees in the Netherlands. Journal of Traumatic Stress, 25, 71-78, e-ISSN: 0894-9867. Recovered from: https://doi.org/10.1002/jts.21665

INEC $(2019 a, b)$. Encuesta Nacional sobre Relaciones Familiares y 
OAI-PMH: http://www.indteca.com/ojs/index.php/Revista_Scientific/oai

Artículo Original / Original Article

Violencia de Género contra las Mujeres - ENVIGMU. Quito, Ecuador: Instituto Nacional de Estadística y Censos. Recuperado de: https://www.ecuadorencifras.gob.ec/documentos/webinec/Estadisticas Sociales/Violencia de genero 2019/Principales\%20 resultados\%20ENVIGMU\%202019.pdf

Lin, N., Dean, A., \& Ensel, W. (1986). Social support, life events, and depression. A. Press, Edition. London, England: Academic Press.

Londoño, N., Henao, G., Puerta, I., Posada, S., Arango, D., \& Aguirre, D. (2006). Propiedades psicométricas y validación de la escala de estrategias de coping modificada (EEC-M) en una muestra colombiana. Universitas Psychologica, 5(2), 327-349, e-ISSN: 16579267. Recuperado de:

https://www.redalyc.org/articulo.oa?id=64750210

Luthar, S., \& Cicchetti, D. (2000). The construct of resilience: Implications for interventions and social policies. Development and Psychopathology, 12(4), 857-885, e-ISSN: 0954-5794. Recovered from: https://doi.org/10.1017/S0954579400004156

Moral de la Rubia, J., López, F., Díaz, R., \& Cienfuegos, Y. (2011). Diferencias de género en afrontamiento y violencia en la pareja. Revista CES Psicología, 4(2), 29-46, e-ISSN: 2011-3080. Recuperado de: https://revistas.ces.edu.co/index.php/psicologia/article/view/1502

Márquez, S. (2006). Estrategias de afrontamiento del estrés en el ámbito deportivo: fundamentos teóricos e instrumentos de evaluación. International Journal of Clinical and Health, 6(2), 359-378, e-ISSN: 1697-2600. Recuperado de:

https://www.redalyc.org/articulo.oa?id=33760209

Naciones Unidas (1995). Informe de la Cuarta Conferencia Mundial sobre la Mujer. ISSN: 92-1-330155-3. A/CONF.177/20/Rev.1. Beijing, China: Publicación de las Naciones Unidas. 
OAI-PMH: http://www.indteca.com/ojs/index.php/Revista_Scientific/oai

Artículo Original / Original Article

OMS (2005). Estudio multipaís de la OMS sobre salud de la mujer y la violencia doméstica: primeros resultados sobre prevalencia, eventos relativos a la salud y respuestas de las mujeres a dicha violencia. ISBN: 92-4-359351-X. Ginebra, Suiza: Organización Mundial de la Salud.

OMS (2002). Informe mundial sobre la violencia y la salud: resumen. ISBN: 92-4-154562-3. Washington, D.C., Estados Unidos: Organización Mundial de la Salud; Organización Panamericana de la Salud.

Rodríguez-Marín, J., Pastor, M., \& López-Roig, S. (1993). Afrontamiento, apoyo social, calidad de vida y enfermedad. Psicothema, 5(Suplem. 1), 349-372, e-ISSN: 0214-9915. Recuperado de:

http://www.psicothema.com/psicothema.asp?id=1148

Torrico, E., Santín, C., Andrés, M., Menéndez, S., \& López, M. (2002). EI modelo ecológico de Bronfrenbrenner como marco teórico de la Psicooncología. Anales de Psicología, 18(1), 45-59, e-ISSN: 02129728. Recuperado de:

https://revistas.um.es/analesps/article/view/28601

UNODC (2010). Handbook on Effective police responses to violence against women. Criminal Justice Handbook Series, ISBN: 978-92-1130291-2. New York, United States: United Nations Office on Drugs and Crime. 


\section{Luís Gabriel Benalcázar Mancero}

e-mail: Igbenalcazar.mpp@uisek.edu.ec

Nacido en Riobamba, Ecuador, 30 de julio del año 1986.

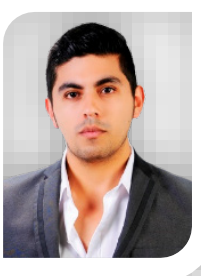

Psicólogo clínico graduado en la Universidad Nacional de Chimborazo (UNACH); he trabajado en varias áreas asociadas al quehacer del profesional en salud mental, dedicándome durante 2 años al trabajo con población vulnerable (discapacidades y adulto mayor) en entidades públicas y privadas; he ejercido la docencia en instituciones de educación superior; también he brindado servicios de asesoría a empresas y he ofrecido atención psicológica en instituciones privadas y benéficas; además del libre ejercicio de mi profesión en la consulta particular. 
OAI-PMH: http://www.indteca.com/ojs/index.php/Revista_Scientific/oai

Artículo Original / Original Article

\section{Paulo César Damián Carrión}

e-mail: pcdamian.mpp@uisek.edu.ec

Nacido en Riobamba, Ecuador, el 31 de enero del año

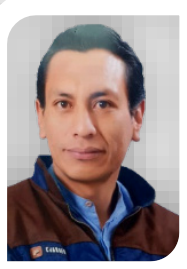
1986. Psicólogo Clínico, graduado en la Universidad Nacional de Chimborazo (UNACH); Dentro de mi trayectoria he trabajado en el Centro especializado CETAD BETESDA, con pacientes con policonsumo de sustancias; he colaborado como Psicólogo Clínico en el Club de futbol profesional Centro Deportivo Olmedo de la ciudad de Riobamba; en la actualidad doy consultas privadas en Psicovida, entidad creada para abordar distintos trastornos psicológicos. 


\section{Artículo Original / Original Article}

\section{Pamela Valeria Yarad Jeada \\ e-mail: vale.yarad@gmail.com}

Nacida en Latacunga, Ecuador, el 18 de febrero del año

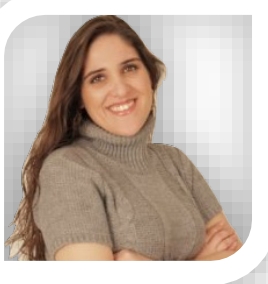
1987. Doctora en Sociología y Antropología; con una maestría en Metodología de la Investigación en Ciencias Sociales; y una Licenciatura en Comunicación Social; La mayor parte de mi carrera, la he desarrollado en España como investigadora y Senior Data Analyst en algunas empresas especializadas en análisis de datos y Social Media; además, me he desempeñado como docente universitaria y he publicado algunos artículos académicos entre los que se destacan: Metodologías móviles: nuevas formas de estudio de lo urbano y Análisis sociológico audiovisual de la publicidad de dispositivos de reproducción móvil digital; mis principales líneas de investigación en las que más me he enfocado son: metodologías de la investigación; social media; sociología del consumo, de la movilidad y de la tecnología; comunicación; cultura y género.

El contenido de este manuscrito se difunde bajo una Licencia de Creative Commons ReconocimientoNoComercial-Compartirlgual 4.0 Internacional 